\title{
Towards the development of a knowledge management practices survey for application in knowledge intensive organisations
}

\author{
C. Steyn \\ Graduate Centre for Management (GCM) \\ Cape Peninsula University of Technology \\ steync@cput.ac.za \\ M. Kahn* \\ Centre for Science, Technology and Innovation Indicators (CeSTII) \\ Knowledge Systems, Human Sciences Research Council \\ Private Bag X9182, Cape Town 8000. Republic of South Africa \\ mkahn@hsrc.ac.za
}

Received January 2008

\begin{abstract}
Various surveys of Knowledge Management Practices (KMPs) in private firms show how an increasing awareness of Knowledge Management (KM) is a critical determinant of an organisation's competitiveness. While private sector strategies favouring internal knowledge sharing and external knowledge protection often provide strategic advantage, public sector research organisations such as South Africa's science councils operate in an environment where, for reasons of wider public interest, transparency is encouraged and knowledge is rendered widely accessible. Unfortunately, however, little has been done to develop rigorous measurements of KMPs in such knowledge intensive organisations (KIOs) that primarily engage in knowledge intensive service activities. To complicate matters further, the majority of studies have been inter-organisational and multi-sectoral, focusing on large organisations in the private sector. Few studies have measured perceptions of KMPs amongst employees of a single organisation and even fewer have focused on KMPs within the public sector. This paper will discuss the theoretical and methodological approach used in the development of a survey aimed at measuring employee perceptions of KMPs within the Human Sciences Research Council (HSRC), one of South Africa's largest public KIOs dedicated to 'social science research that makes a difference.' Principal component analysis of the survey data revealed six factors or constructs applicable to the measurement of KMPs. The results validate the survey instrument and offer a contribution toward the development of a KMP measurement instrument that may be applied across other KIOs in South Africa.
\end{abstract}

*To whom all correspondence should be addressed.

\section{Introduction}

In scanning the KM literature of the last decade, one is bombarded with opinions of skeptics that label it as just the latest management fad. Others question the relevance of analysing a process that has been around since the existence of the modern organisation. Southon and Todd (1999) attribute this growing skepticism to the nature of a large portion of the KM literature, which they describe as "generally popular in style", "highly promotional”, and based on evidence that is largely "anecdotal and case-based with little depth of critical analysis.”

Despite the fact that a clear, over-arching theory of $\mathrm{KM}$ is yet to emerge (Wilson, 2002; Ponzi \& Koenig, 2002), theorists agree that the global business environment is changing in such a way that the management of an organisation's knowledge resources becomes paramount in ensuring competitive advantage (Drucker, 1993; Stewart, 1997). Successful KMPs enable organisations to solve problems and seize opportunities quickly by creating an environment in which relevant, up-to-date strategic knowledge is readily available. As Parlby and Taylor (2000) maintain:

Strategic decision-making depends on predicting what the competition will do or on knowing something better than or ahead of them. The ability to predict market developments and assimilate new ideas is one of the bases of innovation.

According to Sunassee and Sewry (2002: 236), the implementation of an organisation's KM strategy is only likely to contribute to the achievement of organisational goals and outcomes if it is aligned to the overarching business strategy of the organisation. Prior to the development of a KM strategy, managers need to measure the KM practices and/or processes evident in their organisations and how these phenomena relate to each other. This allows one to map the major influences that have an impact on the application of KM (Wiig, 1995), (Henczel, 2000). By so doing, all KM processes and practices must be continually assessed and appraised in terms of the contribution they are able to make to the strategic direction of the organisation. While private sector strategies favouring 
internal knowledge sharing and external knowledge protection often provide strategic advantage, public sector research organisations dedicated to the production of knowledge intensive service activities, such as South Africa's science councils, operate in an environment where, for reasons of wider public interest, transparency is encouraged and knowledge is rendered widely accessible. As a result, the KM strategies implemented within publicly funded research organisations are likely to differ from those implemented in the private sector due to the differing strategic orientations adopted by each.

The nine publicly-funded science research councils of South Africa are knowledge intensive organisations (KIOs) for which poor KM practices may jeopardize their effectiveness through lost institutional memory, knowledge gaps, redundancy of information and misinformed decision making. Furthermore, the pressures these KIOs are experiencing due to declining or static government financial support inevitably bring a more commercial orientation to the provision of research services. Market orientation and competitive-based funding are thus becoming the modus operandi in this sector, the more so given the imperatives of the Public Finance Management Act (RSA, 1999). Coupled with this trend is an increasing demand for transparency about the use of public funds, detection of anti-competitive behaviours and a growing demand for accountability. As a result, science councils are confronted with the challenge of managing their most valuable resources, which are knowledge based, in a more explicit and transparent manner.

Many of these institutions claim to apply KM principles and have appointed a chief knowledge officer or similar at senior level. It was thus felt to be timely to investigate KMPs in one of these KIOs. Unfortunately, however, there is an absence of both qualitative and quantitative research on the state of KMPs in the science council sector. Though these bodies are subject to regular external review, such exercises tend to concentrate on strategic intent and focus on issues such as mandate, human resource development and stakeholder satisfaction. Internal mechanisms for knowledge production and management cannot receive much attention in the rapid evaluations associated with external reviews. Since there has been no comprehensive, large-scale survey of KMPs in the sector, there is no platform on which to base substantive analysis. To further complicate matters, little has been done to develop rigorous measurements of KMPs within organisations, so that Botha (2005:5) notes: “... although much progress has been made during the past decade to develop a philosophy and conceptual framework of KM, the discipline still lacks proven practice.”

The majority of studies that have engaged in the empirical investigation of $\mathrm{KMPs}^{1}$ within organisations have generally embraced the resource-based ${ }^{2}$ view of the firm (Chaung,

\footnotetext{
${ }^{1}$ Actual efforts made at improving $\mathrm{KM}$ practices are defined as arrangements that have been objectively put in place to improve organisations' KM practices, such as organisational arrangements, staff mobility and training, incentives to share knowledge, etc.

${ }^{2}$ The resource-based view of the firm focuses attention on the organisation's ability to mobilize its strategic resources to ensure competitive advantage (Botha, 2005).
}

2004; Marchand, Kettinger \& Rollins, 2002; Alavi \& Leidner, 2002; Botha \& Fouche, 2002; Botha, 2005; Davel \& Snyman, 2005). Other studies have focused on the measurement of KM processes and have concentrated on the analysis of the knowledge creation cycle. Instead of examining organisational practices and the manner in which they contribute to effective KM, these theorists have been primarily concerned with the flows of knowledge into, through and out of the organisation (Wang \& Ariguzo, 2004: 446; Cummings \& Teng, 2003; Chang, Lee, Lee \& Kang, 2004; Choi \& Lee, 2003). Furthermore, the majority of studies aimed at measuring KMPs and processes in the organisation have been inter-organisational and multisectoral, focusing on large organisations in the private sector. Little research has been conducted to measure the views of KMPs amongst employees within an organisation and even fewer have focused on KMPs within the public sector.

Accordingly, in 2005, the Human Sciences Research Council conducted a KMPs Survey aimed at measuring employee perceptions within the organisation. It is hoped that by outlining the theoretical and methodological framework used to develop the survey, this paper will provide a platform upon which further research into the measurement of KMPs within other KIOs may be conducted.

In the sections that ensue the literature on KMPs and their measurement will be reviewed. This will be followed by an exploration of the theoretical and methodological approach to the survey conducted in the HSRC, after which the results of principal component analysis will be investigated in order to evaluate the efficacy of the constructs contained in the survey instrument.

\section{A review of the literature}

An overarching theory of $\mathrm{KM}$ is yet to emerge amidst the broad range of definitions that exist. Maier and Remus (2003) maintain that the primary reasons surrounding the confusion and ambiguity associated with the field are that KM research and scholarly pursuit span a number of disciplinary boundaries and schools of thought. Some view $\mathrm{KM}$ as the management of processes that enable the movement of knowledge into, through and out of the organisation with the ultimate aim of enhancing organisational efficiency and performance (Firestone \& McEllroy, 2005; Darroch, 2003). Others (Wigg, 2000; Nonaka and Takeuchi, 1995) stress that KM encompasses the manipulation of all knowledge related activities, practices, programmes and policies in the organisation with the ultimate aim of applying existing organisational knowledge to create new knowledge. Yet other definitions maintain that the aim of $\mathrm{KM}$ strategies is to facilitate learning and the creation of new knowledge by teaching individuals where to find appropriate organisational knowledge, how to use and apply it effectively and to share and disseminate it appropriately (Mason \& Pauleen, 2003; Senge, Kleiner, Roberts, Ross \& Smith, 1999).

Hendricks and Vriens (1999: 115) capture the essence of a number of definitions by stating that $\mathrm{KM}$ in organisations 
should primarily be concerned with (i) managing the available pool of knowledge and (ii) managing the creation of new knowledge. In managing the available pool of knowledge organisations need to ensure that the right knowledge gets to the right place at the right time; while the creation of new knowledge is dependent on conditions that facilitate organisational learning. For the purposes of this study then, KMPs are defined as an organisational capability that covers any intentional and systematic process or practice of acquiring, capturing, storing, sharing, transmitting and using productive knowledge wherever it resides to enhance learning and performance in organisations (Scarborough, Swan \& Preston, 1999).

KMPs take a variety of forms, thus creating a need for a flexible approach to describing and classifying them. This approach needs to be able to cope with practices that are both formal and informal, paper-based and electronic; people driven and system-driven; wholly or only partially centered on KM. Accordingly a thorough review of available literature on the topic and content analysis of a number of KM surveys was conducted with the aim of identifying KMPs that were particularly relevant within the South African science council sector.

A number of studies attempting to measure KMPs serve as a point of departure. In the South African context Botha (2000, 2005) and Botha and Fouche (2002) developed a data collection and analysis instrument for an annual survey of KMPs in the South African business sector. The model, known as the KM Reference Model, focuses on the interrelationship between organisational culture, structure, processes and technology, which constantly align themselves with organisational leadership and are monitored by numerous organisational measures (Botha, 2005:2). Sunassee and Sewry (2002, 2003) have conducted research on the KM strategies applied by organisations in the vehicle manufacturing industry. Through their research, they have constructed a framework consisting of three interlinked components, namely "KM of the organisation", "KM of the people” and "KM of infrastructure and processes."

The "KM of the organisation" component encompasses all the activities that need to be implemented by the organisation in order for the $\mathrm{KM}$ strategy to prove successful. These include, among others, the creation of an organisational vision that supports KM, the setting of goals and objectives that support a KM strategy and the management of an appropriate organisational culture. The "KM of people” involves managing individual behaviour and expectations during the implementation of a $\mathrm{KM}$ strategy. By so doing, the organisation should encourage knowledge sharing and individual learning and reward these with appropriate incentives. Finally, the "KM of infrastructure and processes" involves adopting collaborative technologies, appropriate knowledge repositories and the implementation of appropriate business processes that have the potential to measure the impact of a KM strategy.

Chaung (2004: 461) developed a survey instrument measuring the relationship between an organisation's KM capability and the organisations overall effectiveness. As a point of departure, Chaung (2004: 460) identified two organisational resources that can effectively be employed in the management of knowledge. Technical KM resources include all physical information technology assets that form part of an organisation's overall information technology infrastructure, such as computer and communication technologies and shareable technical platforms and databases. If correctly employed, technical KM resources can facilitate the rapid collection, storage and exchange of knowledge and integrate fundamental flows of knowledge. Social KM resources, on the other hand, are derived from relationships within the social unit of the organisation and include the organisation's culture, structure and knowledge that various employees of a task or discipline possess.

In a similar study, Alavi and Leidner (2002: 15) invited a non-random sample of 109 participants from 12 different countries to participate in a survey that aimed to assess the meanings that managers ascribe to the concept of KM. When asked what capabilities their organisations needed for successful KM, three general perspectives emerged. Information-based capabilities included the categorization of data, filtered organisational information and people information archives. Technology-based capabilities included integrated databases, larger bandwidth, webproducts, and navigation and retrieval tools; while culturebased capabilities included teamwork, practical organisational guidelines and knowledge sharing.

The sharing of knowledge within KIOs involves a number of challenges. According to Jordan and Jones (1997: 394) the extent of knowledge sharing in organisations is largely dependent on the nature of knowledge ownership that exists within the organisation. For instance, when an individual regards their knowledge as being part of their identity within the organisation, they may be less willing to share this knowledge freely within the organisation. Furthermore, in organisations where business processes or units are largely specialized, knowledge may become highly situated, or "sticky" due to a lack of common semantics amongst business units through which to share such knowledge. By their very nature, KIOs focusing primarily on research activities are comprised of highly specialized individuals, who, by virtue of their professions, rely on knowledge as a source of professional identity. As a result, such KIOs are particularly prone to the challenges of knowledge sharing discussed above.

\section{Theoretical framework}

In order to design the survey instrument further review of the literature was undertaken around the five broad organizational components of organisational culture, information and communication technologies (ICTs), human resource management (HRM) practices, organisational leadership and organisational structure.

A brief discussion of each component and its associated practices follows. 


\section{Organisational culture}

Almost all approaches to KM regard organisational culture as a key component of any effective KM strategy. According to Rollet (2003: 24), organisational culture can either drive or inhibit an organisation's KM strategy and is probably also the most difficult organisational component to control. Similarly, Ndlela and Du Toit (2000 as cited in Davel \& Snyman, 2005) maintain that the types of culture present in an organisation affect the way in which knowledge is managed, and can, as a result, either persuade or discourage the use of KM practices. Similarly, Rollet (2003) maintains that organisational cultures appropriate to effective KM strategies dispel fears that sharing knowledge may result in a loss of power in the organisation. Healthy conflict should be encouraged and the importance of experimentation should not be underestimated.

As mentioned previously, KIOs, by their very nature, are particularly prone to challenges surrounding knowledge sharing. Marchand, Kettinger and Rollins (2002:103) identify two aspects of organisational culture that are of particular importance in knowledge intensive organisations, namely "transparency" and "knowledge sharing". "Transparency", refers to an environment where errors, mistakes and failures are shared and used as learning opportunities. For an environment to be transparent, employees must trust each other and, as a result, feel free to experiment and explore. Although a degree of internal competition can be healthy in certain organisational environments, high levels of internal competition can lead to lower levels of trust and a subsequent decline in organisational transparency. For the purposes of the HSRC survey, organisational culture was modeled as a second order construct that was measured by the first-order constructs of transparency and knowledge sharing.

\section{Information and communication technologies (ICTs)}

According to Skyrme (1998: 73) ICTs enable the knowledge creation process through the conversion of knowledge from inputs to outputs. ICTs enhance knowledge inputs by condensing, filtering and presenting data, processing that data, storing it, facilitating its flow through the organisation and finally supporting the thinking processes that inform effective decision-making. Marchand, Kettinger and Rollins (2002: 53) make an elaborate distinction between information technology for management support, innovation support, business process support and operational support. For the purposes of the current research, a distinction between the various kinds of ICT support was deemed inappropriate, since the survey was designed for application across a number of functional areas and ranks within a single organisation.

\section{Human resource management (HRM) practices}

Although ICTs can greatly enable effective KM, the willingness of people in organisations to share information can prove to be a critical constraint to the implementation of KMPs. As a result, the political aspects of social exchange should be considered when implementing a KM strategy in any organisation. Proponents of the learning organisation approach emphasize the importance of tacit knowledge that is largely embedded in work practices (Currie \& Kerrin, 2003: 1027). They recognise that sophisticated tools are unable to capture sufficiently contextualised knowledge and argue in favour of the establishment of communities of practice within organisations that enable individuals to identify others with potential resources and reach out to them to seek out these resources. Conditions of trust, shared norms, values, obligations and expectations, common content and language are crucial to the establishment of such networks. In this instance, the HRM function can play a crucial role in enabling KMPs by creating and maintaining an organisational climate conducive to such conditions. According to Currie and Kerrin (2003: 1030) such networks of relationships constitute an important form of social capital, which is crucial to effective knowledge sharing in organisations. Similarly, Scarborough and Carter (2000:66) suggest that HRM practices can best contribute to managing knowledge by influencing behavioural responses from employees and the stock of human and social capital that is available to the firm. Through practices such as performance management, career structuring, recruitment and selection, career management and organisational development an environment conducive to knowledge acquisition, utilization and sharing can be created.

\section{Organisational structure}

Paul Meyers (1996:2) regards organisational design as one of the key enablers to successful KM. Organisational design encompasses elements of an organisation's structure and includes the division of labour, the allocation of decision rights, the delineation of organisational boundaries and networks of informal relationships. Similarly, Pinchot and Pinchot (1996: 49) maintain that in order for knowledge to be utilised effectively in the knowledge economy, organisations will have to make a number of fundamental shifts in terms of organisational structure. These shifts include a move from individual work to team work, from functional work to project-based work; from single-skilled personnel to multi-skilled employees and from co-ordination from above to co-ordination among peers. Matrix organisation structures, where mid-level employees report to both a project managers and a functional managers have been shown to improve structural integration in the organisation and improve overall creativity and innovation (Moss-Kanter, 1996: 96)

\section{Leadership}

Organisational management and leadership can also influence the outcome of a KM strategy by influencing the nature of knowledge resources present in the organisation, their deployment and their utilization (Sunassee \& Sewry, 2002). To ensure the successful implementation of a KM strategy, management should ideally create the conditions that cultivate employee acquisition and use of KM skills by enabling convenient access to the needed knowledge resources in the organisation. Managers are also responsible for the proper co-ordination of an organisation's activities by aligning employees' knowledge with the organisational strategy; allocation the appropriate financial resources and assigning staff to infrastructural roles. Management can 
influence the outcome of a KM strategy by installing the mechanisms necessary for measuring and evaluating organisational resources and KM activities.

Appropriate items measuring the various constructs discussed above were developed and included in the survey (Refer to Table's I and II for a list of items used).

\section{Research methodology}

The Centre for Science, Technology and Innovation Indicators (CeSTII) already performs two surveys that relate to intellectual capital production, namely the annual Research \& Experimental Development Survey that follows the Frascati Guidelines (OECD, 2002) and the Innovation Survey that follows the Oslo Manual Guidelines (OECD, 2005). It therefore seemed logical for CeSTII to conduct the KMPs Survey as another step toward understanding the intellectual capital black box. In designing the survey instrument extensive use was made of two international studies namely the OECD and Statistics Canada's Survey of KM Practices in the Business Sector (2003) and the OECD's (2002) Survey on KM Practices for Ministries/Departments/Agencies of Central Government in OECD Member Countries. The former survey focused on the use of 23 KMPs used by private sector firms and included questions relating to the incentives for using $\mathrm{KM}$ practices and the results associated with implementing KM practices. The Public Services questionnaire targeted respondents at director level and focused on practices that facilitate personnel development and mobility, the transfer of competencies amongst employees and business units, information management policies and strategies, KM budgets etc.

Given the range of KM resources or capabilities that could be incorporated into a survey of such a nature and the potential burden on respondents resulting from too many items, it was decided to focus on those $\mathrm{KM}$ practices that would ultimately facilitate the achievement of the strategic objectives of a KIO such as the HSRC. As a KIO focused on rendering knowledge intensive services, the HSRC employs researchers to create and disseminate knowledge according to the vision statement 'social science that makes a difference.' As a result, the organisation finds itself operating across a number of domains: public goods research; as contractor; as consultant; and as policy advisor. Across these diverse roles the management of intellectual capital is of critical importance to the HSRC, especially since this is a commonly cited source of competitive advantage in the marketplace. This is particularly so given the increasing emphasis on a business orientation in the organisation, the development of new technology-based methods for the creation, storage and distribution of knowledge, and the fundamental impact of internet technologies upon traditional academic knowledge linkages and networks and the creation of new knowledge cultures. Since the primary objective of the HSRC is the creation of new knowledge through research, practices associated with the collection, utilization and sharing of knowledge were regarded as of primary importance for inclusion in the survey.
Following our literature review twenty items representing the five broad organisational design components were included in the instrument.

\section{Questionnaire design}

The questionnaire was designed to capture the extent to which respondents believed that the various KMPs associated with the components mentioned in the previous section were evident within the HSRC. The survey consisted of four sections that captured background information, information needs and usage in the HSRC, perceptions of organisation culture and the HSRC knowledge context.

A Reference Committee consisting of both HSRC and external experts was established to oversee the design and implementation of the survey. The questionnaire was piloted amongst a selected sample of HSRC staff representative of both function and rank. A method of cognitive interviewing was employed with the objective to elicit feedback from respondents with regards to question intent, the meaning of terms, recallability of information and response processes. Changes to the questionnaire were made following the completion of a first pilot, after which a second pilot was conducted. The second pilot, which took the form of a pretest was conducted in order to test the variability within the population to be surveyed and the adequacy of the questionnaire. Once again, pilot respondents were selected to represent both function and rank.

\section{Data collection}

Prior to the launch of the survey, an HSRC wide communication strategy was launched. Approximately one month before the launch of the survey, information about the survey was posted on the HSRC Intranet, accompanied by a series of frequently asked questions. Two weeks before the launch of the survey a poster series was displayed throughout the HSRC buildings in Cape Town, Pretoria and Durban. The aim of the communication strategy was to:

1) Create awareness of the survey in order to ensure the highest possible return rate

2) Educate employees on the importance of KM within the HSRC

A self-administered electronic questionnaire was e-mailed to all HSRC employees. Employees that did not have access to computers were identified and given hard-copy questionnaires to complete. In both instances, complete confidentiality and anonymity were guaranteed. Respondents were initially given one week to complete and submit the questionnaire, but this was eventually extended to two weeks following a low response rate during the first week. Regular reminders were sent out to improve response rates.

On implementation of the survey, the HSRC had 292 staff members that were either permanently appointed, or on a fixed term contract for longer than a year. Of these, 140 were administrative (of which 65 percent were with corporate services, while 35 percent worked in a research 
program) and 152 were research staff. Approximately 117 employees on short-term contracts for less than a year were also surveyed. These consisted mainly of administrative staff, fieldworkers, consultants and call-centre operators. Upon survey close-out, 158 employees had responded, representing a response rate of 32 percent and 53 percent for permanent/long-term contract staff and short-term contract staff respectively. Amongst the permanent/long-term contract staff, a response rate of 33 percent and 32 percent was reached for administrative and research staff respectively. Unfortunately short term contract staff could not be classified according to function.

\section{Data analysis}

According to Schwab (1980) the assessment of construct validity plays an integral part of confirming the adequacy of measures by establishing the degree to which empirical indicators measure the construct under investigation. In other words, to what extent do the items included in the questionnaire measure the underlying constructs associated with perceptions of KMPs? The construct validity (Schwab, 1980) of the measurement instrument discussed in this paper was assessed through the use of principal component analysis with orthogonal rotation ${ }^{3}$.

A general rule of thumb suggests that for principal component analysis there should be ten to twenty-five times as many observations as there are variables in the analysis. This criterion was met for our sample size of 158 employees. Since no significant differences were detected by groups defined by function, position, length of service and type of employment contract, the full sample of 158 employees was used in the analysis. Once missing variables and "Don't Know" responses were excluded, 135 observations were available for analysis.

Tables 1 and 2 present the results of the principal component analysis conducted on the various items. Due to the distinct nature of the items assigned to the construct separate analyses were performed on items relating to organisational culture. Only eigen values greater than one were selected and factors loading less than 0.4 were suppressed. Nunally (1978) recommends the inclusion of factor loadings greater than 0.5 , although many researchers regard factor loadings of 0.3 as sufficient (Hair, Anderson, Tatham \& Black, 1992), (Pedhazur \& Schmelkin, 1991). Due to the limited number of items included in the analysis, factor loadings of 0.4 were regarded as sufficient for the current study.

Table 1 presents the results of the principal component analysis for organisational culture. The analysis presents a Kaiser-Meyer Olkin score of 0.640 , which suggests that the sample used in the analyses proved adequate ${ }^{4}$ for the procedure. Items included in the analysis loaded on two factors, corresponding to the original conceptualizations of knowledge sharing and transparency. Both factors

\footnotetext{
${ }^{3}$ Orthogonal rotation does not permit the latent variables to correlate.

${ }^{4}$ Field (2000:447) regards as Kaiser-Meyer Olkin Score over 0.5 as adequate)
}

displayed reasonable levels of reliability. According to Nunally (1967), alpha values as low as 0.5 are acceptable for the early stages of research. Knowledge Sharing displayed a Cronbach's Alpha of 0.788, while Organisational Transparency displayed a Cronbach's Alpha of 0.667 . The results suggest that the items are adequate measures of the variables they were intended to measure.

\section{Table 1: Principle component analysis - organisational culture}

\begin{tabular}{|c|c|c|}
\hline \multicolumn{3}{|c|}{$\begin{array}{l}\text { Principal Component Analysis of Research Variables N=135 } \\
\text { Kaiser-Meyer-Olkin Measure of Sampling Adequacy }=0.640\end{array}$} \\
\hline Factors and Item Descriptions & $\begin{array}{l}\text { Factor } \\
1\end{array}$ & $\begin{array}{l}\text { Factor } \\
2\end{array}$ \\
\hline \multicolumn{3}{|c|}{ Knowledge Sharing (Cronbach’s Alpha = 0.788) } \\
\hline $\begin{array}{l}\begin{array}{l}\text { Employees are resistant to share their } \\
\text { knowledge }\end{array} \\
\end{array}$ & 0,909 & \\
\hline Employees keep information to themselves & 0,899 & \\
\hline \multicolumn{3}{|c|}{ Organisational Transparency (Cronbach’s Alpha $=0.667$ ) } \\
\hline Employees trust each other & & 0,777 \\
\hline $\begin{array}{l}\text { Employees feel confident to experiment and } \\
\text { explore }\end{array}$ & & 0,773 \\
\hline $\begin{array}{l}\text { Information about failures, mistakes and } \\
\text { errors is openly shared }\end{array}$ & & 0,694 \\
\hline A culture of competitions exists & & 0,501 \\
\hline
\end{tabular}

Table 2 presents the results of the principal component analysis performed on items measuring perceptions of KM practices in the HSRC. The analyses presented a KaiserMeyer-Olkin score of 0.721, rendering the sample adequate for the analysis. Contrary to expectations, the items included in the analysis loaded on four factors, corresponding to ICTs, organisational leadership, organisational structure and design and training. During the conceptualization phase of the research it was assumed that items relating to mentorship and the sharing of knowledge between experienced and inexperienced employees would cluster under a factor relating to HRM Practices. Instead, the sharing of knowledge between experienced and less experienced employees was associated more strongly with organisational leadership behaviours, while mentoring practices clustered with collaborative organisational structures. A fourth factor, not initially considered, emerged and was appropriately labeled Training. All factors presented in Table 2 display reasonable levels of reliability, with Cronbach Alpha scores ranging from 0.583 to 0.788 .

\section{Conclusion}

The paper set out to discuss the theoretical and methodological approach used in the development of a survey aimed at measuring employee perceptions of KMP's within the HSRC with the aim of making a contribution to the development of a KMPs measurement instrument that may be applied across other KIOs in South Africa. Since there has been no comprehensive survey of KMPS in the sector and since most studies that have engaged in the empirical investigation of KMPS have been interorganisational, multi-sectoral and have focused on large organisations in the private sector, it is hoped that the paper has provided a platform upon which further research and 
analysis can be conducted. The survey instrument was constructed following a thorough review and content analysis of empirical studies aimed at measuring $\mathrm{KM}$ in organisations. The survey was successfully applied across all functional groupings within the HSRC, and upon further analysis, no significant differences could be detected between group means broken down by function and position. Principal component analysis revealed six factors or constructs applicable to the measurement of $\mathrm{KM}$ practices:

\section{Table 2: Principle component analysis - organisational practices}

\begin{tabular}{|c|c|c|c|c|}
\hline \multicolumn{5}{|l|}{$\begin{array}{l}\text { Principal Component Analysis of Research Variables N=135 } \\
\text { Kaiser-Meyer-Olkin Measure of Sampling Adequacy }=0.721\end{array}$} \\
\hline Factors and Item Descriptions & $\begin{array}{l}\text { Factor } \\
1\end{array}$ & $\begin{array}{l}\text { Factor } \\
2\end{array}$ & $\begin{array}{l}\text { Factor } \\
3\end{array}$ & $\begin{array}{l}\text { Factor } \\
4\end{array}$ \\
\hline \multicolumn{5}{|l|}{ Information and Communication Technology (Cronbach’s Alpha=0.727) } \\
\hline Sufficient investments in ICT & 0,792 & & & \\
\hline $\begin{array}{l}\text { Sufficient resources are dedicated to detecting and obtaining information from external } \\
\text { organisations }\end{array}$ & 0,704 & & & \\
\hline $\begin{array}{l}\text { Sufficient resources are dedicated to detecting and obtaining information from external } \\
\text { organisations }\end{array}$ & 0,653 & & & \\
\hline The ICT systems at my disposal are sufficient to support my work & 0,601 & & & \\
\hline \multicolumn{5}{|l|}{ Organisational Leadership (Cronbach’s Alpha $=0,727$ ) } \\
\hline Managers regularly involve staff in decision-making & & 0,857 & & \\
\hline Managers consult with staff on a regular basis & & 0,831 & & \\
\hline Experienced employees share their experiences with less experienced employees & & 0,603 & & \\
\hline \multicolumn{5}{|l|}{ Collaborative Structures (Cronbach Alpha $=0,600)$} \\
\hline Staff interact informally with staff from other units & & & 0,705 & \\
\hline The exchange of information across research programs and business units is facilitated & & & 0,685 & \\
\hline Research and/or business units are encouraged to collaborate with each other & & & 0,637 & \\
\hline I have received mentoring from other employees & & & 0,600 & \\
\hline \multicolumn{5}{|l|}{ Training (Cronbach’s Alpha $=0,583$ ) } \\
\hline My skills are sufficient to source information necessary to my work & & & & 0,816 \\
\hline I have received sufficient training on information and communication technologies & & & & 0,740 \\
\hline I have received sufficient training to keep my skills current & & & & 0,415 \\
\hline
\end{tabular}

- Cultural dimensions of knowledge sharing

- $\quad$ Organisational transparency

- Information and communication technologies

- $\quad$ Organisational leadership

- Collaborative organisational structures and

- $\quad$ Training

The six constructs or factors listed above correspond closely to the organisational design components used to develop the instrument items. At the outset of the project, HRM was conceptualized as a key organisational practice that would contribute to the management of knowledge in the HSRC. The results of the principal component analysis, however, revealed a construct that was more closely related to organisational training - a mere component of the HRM function. While the results of the research validate the survey instrument and its application, future research efforts could focus on expanding the concept and measurement of $\mathrm{HRM}$ as it pertains to KM in KIOs.

Further analysis, using the constructs derived from the principal component analysis, revealed a moderate level of knowledge sharing and low levels of transparency amongst HSRC employees. Given the nature of academic work, where knowledge forms an integral part of one's professional development, a certain degree of knowledge "stickiness" is to be expected. Willingness to part with this kind of knowledge will largely depend on the extent to which the individual will be rewarded or valued for sharing such knowledge. Given the importance of the knowledge sharing and transparency in KIOs, future research attempts could also consider expanding the measurement of these concepts by linking them to issues of knowledge identity and ownership.

\section{Acknowledgements}

The authors wish to thank the following people for their assistance with the survey:

Dr. Sagren Moodley, Ms. Simone Esau, Mr. Stanley Ntakumba, Mr. Nkhosiko Batatu (HSRC Research Team); Ms. Faye Reagon (Reference Committee - HSRC), Dr. Andrew Paterson (Reference Committee - HSRC), Mr. Daan Botha (Reference Committee - University of Stellenbosch)

\section{References}

Alavi, M. \& Leidner, D. 2002. 'KM Systems: Issues, challenges and benefits.' In Barnes, S. (Ed.). KM Systems: Theory and practice. Oxford: Alden Press.

Botha, D.F. 2000. 'A conceptual framework for the management of knowledge in a knowledge based 
enterprise', South African Journal of Business Management, 31(4): 141-148.

Botha, D.F. 2005. 'Towards an instrument for surveying KM Practices', South African Journal of Business Management, 36(1): 1-6.

Botha, D.F. \& Fouche, B. 2002. 'KM practices in the South African business sector: Preliminary findings of a longitudinal study', South African Journal of Business Management, 33(2): 13-19.

Chang, K., Lee, K., Lee, S. \& Kang, I. 2004. 'KMPI: Measuring KM performance', Information and Management, 42: 469-482.

Chaung, S. 2004. 'A resource-based perspective on KM capability and competitive advantage: An empirical investigation', Expert Systems with Applications, 27(3): 459-465.

Choi, B. \& Lee, H. 2003. 'An empirical investigation of KM styles and their effect on corporate performance', Information and Management, 40: 403-417.

Cummings, J.L. \& Teng, B. 2003. 'Transferring R\&D knowledge: The key factors affecting knowledge transfer success', Journal of Engineering and Technology Management, 20: 39-68.

Currie, G. \& Kerrin, M. 2003. 'Human resource management and KM: Enhancing knowledge sharing in a pharmaceutical company', International Journal of Human Resource Management, 14(6): 1027-1045.

Darroch, J. 2003. 'Developing a measure of KM practices and behaviours', Journal of KM, 7(5): 41-54.

Davel, R. \& Snyman, M. 2005. 'Influence of corporate culture on the use of KM techniques and technologies', South African Journal of Information Management, 7(2).

Drucker, P. 1993. Post Capitalist Society. Oxford: Butterworth-Heinmann.

Field, A.P. 2000. Discovering statistics using SPSS for windows: Advanced techniques for the beginner. London: Sage.

Firestone, J.M. \& McElroy, M.W. 2005. 'Doing KM', The Learning Organisation, 12(5): 189-212.

Hair, J., Anderson, R., Tatham, R. \& Black, W. 1992. Multivariate data analysis. 3rd Edition. New York: Macmillan.

Henczel, S. 2000. 'The information audit as a first step towards effective KM: An opportunity for the specialist librarian', Inspel, 34(3/4): 210-226.

Hendricks, P.H.J. \& Vriens, D. 1999. 'Knowledge-based systems and KM: Friends or foes?', Information and Management, 35: 113-125.
Jordan, J. \& Jones, P. 1997. ‘Assessing your company’s KM style’, Long Range Planning, 30(3): 392-398.

Marchand, D.A., Kettinger, W.J. \& Rollins, J.D. 2002. Information orientation: The link to business performance. Oxford: Oxford University Press.

Mason, D. \& Pauleen, D.J. 2003. 'Perceptions of KM: A qualitative analysis', Journal of KM, 7(4): 38-48.

Maier, R. \& Remus, U. 2003. 'Implementing processoriented KM strategies', Journal of KM, 7(4): 62-74.

Moss-Kanter, R. 1996. 'When a thousand flowers bloom: Structural, collective and social conditions for innovation in organisations.” In Myers, P. (Ed.). KM and organisational design. Newton, Massachusetts: Butterworth Heinemann.

Myers, P. 1996. 'KM and organisational design: An introduction.' In Myers, P. (Ed.). KM and organisational design. Newton, Massachusetts: Butterworth Heinemann.

Nonaka, I. \& Takeuchi, H. 1995. The knowledge creating company. Oxford: Oxford University Press.

Nunally, J. 1967. Psychoanalytic theory, New York: McGraw Hill.

Organisation for Economic Cooperation and Development (OECD). 2002. Frascati Manual: Proposed standard practice for surveys on research and experimental development. Paris: OECD.

Organisation for Economic Cooperation and Development (OECD). 2005. Oslo Manual: The measurement of scientific and technological activities. Paris: OECD.

Organisation for Economic Cooperation and Development (OECD) Public Management Service Directorate. 2002. Survey on KM practices: For ministries/departments/agencies of central government in OECD member countries. Paris: OECD.

Organisation for Economic Cooperation and Development (OECD) and Statistics Canada. 2003. Measuring KM in the business sector: First steps. Paris: OECD/Statistics Canada.

Parlby, D. \& Taylor, R. 2000. The power of knowledge: A business guide to KM. [Online] URL:http://www.kpmgconsulting.com

Pedhazur, E. \& Schmelkin, L. 1991. Measurement, design and analysis: An integrated approach. New Jersey: Lawrence Erlbaum Associates.

Pinchot, G. \& Pinchot, E. 1996. 'The rise and fall of bureaucracy.' In Myers, P. (Ed.). KM and organisational design. Newton, Massachusetts: Butterworth Heinemann.

Ponzi, C. \& Koenig, M. 2002. 'KM: Another management fad?', Information Research, 8(1). 
Rollet, H. 2003. KM: Processes and technologies. Massachusetts: Kluwer Academic Publishers.

RSA. 1999. Public Finance Management Act No. 1 of 1999 . Pretoria: Republic of South Africa

Scarborough, H. \& Carter, C. 2000. Investigating KM. London: CIPD.

Scarborough, H., Swan, J., \& Preston, J. 1999. KM: A literature review. London: Institute of Personnel and Development.

Schwab, D. 1980. 'Construct validity in organisational behaviour’, Organisational Behaviour, 2:3-43.

Senge, P., Kleiner, A., Roberts, C., Ross, R.G. \& Smith, B. 1999. The dance of change: The challenges to sustaining momentum in learning organisations. New York: Doubleday.

Skyrme, D. 1998. Measuring the value of knowledge: Metrics for the knowledge-based business. London: Business Intelligence Ltd.

Southon, G. \& Todd, R. 1999. 'KM: A social perspective'. Paper presented at the KNOW Conference, Sydney, November 1999.

Stewart, T.A. 1997. Intellectual capital: the new wealth of organizations. New York: Doubleday.

Sunassee, N.N. \& Sewry, D.A. 2002. 'A theoretical framework for knowledge management implementation.' In Kotze, P., Venter, L. \& Barrow, M (Eds.). Enablement through technology. Proceedings of SAICSIT 2002, Annual Research Conference of the South African Institute of Computer Scientists and Information Technologists. Pretoria: SAICSIT.

Sunassee, N.N. \& Sewry, D.A. 2003. 'An investigation of knowledge management implementation strategies.' In Eloff, J., Kotze, P., Engelbrecht, A. \& Eloff, M. (Eds.). IT research in developing countries. Proceedings of SAICSIT 2003, Annual Research Conference of the South African Institute of Computer Scientists and Information Technologists. Pretoria: SAICSIT.

Wang, S. \& Arigyzo, G. 2004. ' $\mathrm{KM}$ through the development of information schema', Information and Management, 41: 445-465.

Wigg, K.M. 2000. 'KM: An emerging discipline rooted in a long history', In Dupres, C. \& Chauvel, D. (Eds.). Knowledge horizons. Boston: Butterworth-Heinemann.

Wiig. K.M. 1995. KM methods: Practical approaches to managing knowledge. Arlington, TX: Schema Press.

Wilson, T.D. 2002. 'The nonsense of KM', Information Research, 8(1). [Online] URL:

http://informationr net/ir/8-1/paper144 html. 